\title{
BMJ Open Biofortification of wheat with zinc for eliminating deficiency in Pakistan: study protocol for a cluster-randomised, double-blind, controlled effectiveness study (BIZIFED2)
}

\author{
Nicola M Lowe (D) , ${ }^{1}$ Mukhtiar Zaman, ${ }^{2}$ Victoria Hall Moran (D) , ${ }^{1}$ Heather Ohly, \\ Jonathan Sinclair, ${ }^{1}$ Sadia Fatima, ${ }^{3}$ Martin R Broadley, ${ }^{4}$ Edward J M Joy, ${ }^{5}$ \\ Usman Mahboob, ${ }^{6}$ R Murray Lark, ${ }^{4}$ Munir H Zia, ${ }^{7}$ E Louise Ander, ${ }^{8}$ Paul A Sharp, ${ }^{9}$ \\ Elizabeth H Bailey, ${ }^{4}$ Scott D Young, ${ }^{4}$ Muhammad Jaffar Khan ${ }^{3}$
}

To cite: Lowe NM, Zaman M, Moran VH, et al. Biofortification of wheat with zinc for eliminating deficiency in Pakistan: study protocol for a cluster-randomised, doubleblind, controlled effectiveness study (BIZIFED2). BMJ Open 2020;10:e039231. doi:10.1136/ bmjopen-2020-039231

- Prepublication history for this paper is available online. To view these files, please visit the journal online ().

June 2019

Received 15 April 2020

Revised 30 September 2020 Accepted 23 0ctober 2020

Check for updates

(c) Author(s) (or their employer(s)) 2020. Re-use permitted under CC BY-NC. No commercial re-use. See rights and permissions. Published by BMJ.

For numbered affiliations see end of article.

Correspondence to Professor Nicola M Lowe; NMLowe@uclan.ac.uk

\section{ABSTRACT}

Introduction Micronutrient deficiencies, commonly referred to as 'hidden hunger', affect more than two billion people worldwide, with zinc and iron-deficiency frequently reported. The aim of this study is to examine the impact of consuming zinc biofortified flour (Zincol-2016) on biochemical and functional measures of status in adolescent girls and children living in a low-resource setting in Pakistan.

Methods and analysis We are conducting a pragmatic, cluster-randomised, double-blind, controlled trial. A total of 482 households have been recruited from two catchment areas approximately $30-40 \mathrm{~km}$ distance from Peshawar. Household inclusion criteria are the presence of both an adolescent girl, aged 10-16 years, and a child aged $1-5$ years. The study duration is 12 months, divided into two 6-month phases. During phase 1, all households will be provided with locally procured flour from standard varieties of wheat. During phase 2, clusters will be paired, and randomised to either the control or intervention arm of the study. The intervention arm will be provided with zinc biofortified wheat flour, with a target zinc concentration of $40 \mathrm{mg} / \mathrm{kg}$. The control arm will be provided with locally procured wheat flour from standard varieties with an expected zinc concentration of $20 \mathrm{mg} / \mathrm{kg}$. The primary outcome measure is plasma zinc concentration. Secondary outcomes include anthropometric measurements, biomarkers of iron and zinc status, and the presence and duration of respiratory tract infections and diarrhoea.

Ethics and dissemination Ethical approval was granted from the University of Central Lancashire STEMH Ethics Committee (reference number: STEMH 1014) and Khyber Medical University Ethics Committee (DIR/KMU-EB/ BZ/000683). The final study methods will be published in peer-reviewed journals, alongside the study outcomes. In addition, findings will be disseminated to the scientific community via conference presentations and abstracts and communicated to the study participants through the village elders at an appropriate community forum. Trial registration number ISRCTN17107812; Pre-results.
Strengths and limitations of this study

- The study will build on and scale up our previous Biofortified Zinc Flour to Eliminate Deficiency trial, with a focus on adolescent girls and children, and will include both biochemical and functional biomarkers of zinc status.

- Iron status will also be monitored as a secondary outcome measure because our previous study revealed that the Zincol-2016 biofortified wheat has greater iron and zinc concentrations compared with control grain.

- The Zincol-2016 used in the effectiveness study will be grown by local farmers, therefore, giving a realistic picture of what is possible if the distribution of the grain was scaled up.

- A limitation is that, since designing the study, a flour fortification programme has been initiated in this region and some of the locally purchased flour may be fortified with iron, zinc and folate, although this will not impact on our primary outcome measure.

\section{INTRODUCTION}

Micronutrient deficiency, commonly referred to as 'hidden hunger', affects more than two billion people worldwide, with the highest burden occurring in low-income and middleincome countries (LMICs). ${ }^{1}$ Zinc and iron are two of most frequently reported micronutrient deficiencies, due in part to their low bioavailability from plant food sources which make up most of the calorie intake in lowresource communities. In Pakistan, the recent National Nutrition Survey (NNS) reported that $22.1 \%$ of women of reproductive age (WRA) and $18.6 \%$ of children under 5 years of age are zinc deficient. ${ }^{2}$ Although this is an improvement since the last NNS survey in 
2011, where the values were $41.9 \%$ and $36.5 \%$, respectively, the national survey headline does not reflect the situation in some of the most marginalised communities. Results from our own research focusing on communities living in the brick kiln areas around Peshawar revealed that the prevalence of zinc deficiency among WRA is $30 \%$, based on the prevalence of plasma zinc concentration below $660 \mu \mathrm{g} / \mathrm{L} .{ }^{3}$ The health consequences of zinc deficiency include greater risk of stunted growth and impaired neurodevelopment in children, increased susceptibility to infections in children and adults, and complications during the pregnancy and childbirth. ${ }^{3}$ Diarrhoea is a major cause of morbidity and mortality in children under 5 years in LMICs, and both upper respiratory tract infections and diarrhoea respond positively to zinc supplementation. ${ }^{4}$

According to the NNS, iron-deficiency anaemia (IDA) affects $18.2 \%$ of WRA and $28.6 \%$ of children $<5$ years. This is a modest improvement since 2011 (19.9\% and 33.4\%, respectively). ${ }^{2}$ IDA affects maternal and child mortality, physical and cognitive development in children and work productivity in adults. ${ }^{5}$

The Food Fortification Programme (FFP) in Pakistan was launched in 2016 and seeks to provide support to industry to adequately fortify wheat flour with iron and folate, and edible oil/ghee with vitamin A. The FFP is also supporting government capacity and infrastructure for laboratory testing, so that fortification is conducted to meet quality standards. This approach is undoubtedly an important part of the solution to hidden hunger in Pakistan; however, fortification of wheat flour will only reach those who purchase flour from mills participating in this programme. Over $60 \%$ of Pakistan's wheat harvest is retained by farmers for self-consumption or used as in-kind payments to labourers. ${ }^{6}$ Most of this wheat is milled locally and not in the large industrial mills where flour fortification occurs.

An increasing body of evidence suggests that biofortification, a process by which the nutritional quality of food crops is enhanced through plant breeding and/or agronomic practices, may be a cost-effective and sustainable approach to reducing micronutrient deficiency, and may complement other strategies including fortification at processing stage and dietary diversification. A key challenge here is to assess the impact of biofortification in the context of spatially variable soils, with different concentrations of plant-available zinc; differences in basic soil properties that affect zinc uptake and in crops that are subject to differing management strategies, given the variations in resource endowment of farmers and in their environment.

Biofortified Zinc Flour to Eliminate Deficiency (BIZIFED2) follows on from our recently completed project, BiZiFED: (May 2017-April 2019). BiZiFED was a Foundation Award, funded by the Biotechnology and Biological Sciences Research Council (BBSRC) under the Global Challenges Research Fund (BB/P02338X/1). ${ }^{78}$ BIZIFED2 will build on our previous study by exploring the impact of consuming flour milled from biofortified wheat grain (Zincol-2016) on biochemical and functional indices of zinc and iron status in adolescent girls and children living in a low-resource setting in North West Pakistan. Adolescence is a critical time in the life course for physical and psychosocial development and attention to adolescent girls' nutrition is an important preventive strategy that has the potential to improve adolescent girls' health, including cognitive function, maternal and neonatal outcomes and the future health of their offspring. Investing in the health and development of adolescents is, therefore, essential for achieving the Sustainable Development Goals, promoting public health and ensuring economic development.

The objective of this study is to examine the impact of consuming zinc biofortified flour on biochemical and functional measures of zinc status in adolescent girls and children. Secondary objectives are to quantify the contribution to total dietary zinc and iron from biofortified flour, the impact of consuming the biofortified flour on iron status and to examine the bioavailability of zinc and iron from the flour.

\section{METHODS AND ANALYSIS}

\section{Study setting and recruitment}

The study is taking place in Khyber Pakhtunkhwa (KP) Province. There are two neighbouring catchment areas, A $\left(5 \mathrm{~km}^{2}\right)$ and B $\left(4.5 \mathrm{~km}^{2}\right)$, located $30-40 \mathrm{~km}$ south east of Peshawar. Area A was the location of our previous study, and a good working relationship with this community has already been established, along with a detailed knowledge of the nutritional status of the WRA. Area B has not been involved in previous research conducted by this team, however, a good relationship with our implementation partner, Abaseen Foundation Pakistan (AFPK) was already in place as a result of an established AFPK education project. Cocreation of the research questions and dissemination of findings is embedded within the long-term relationship between the project partners and achieved through ongoing engagement with the community and Jirga (respected male elders) and in accordance with the Global Code of Conduct. ${ }^{9}$ In order to assess the willingness and suitability of Area B as a catchment area for this study, a consultation was undertaken with the community Jirga where the purpose of the study was explained and discussed. With the Jirga's permission, a pilot study of 50 WRA living in households within this area was undertaken to assess their dietary patterns (macro and micronutrient intakes), zinc status and the mineral content of the ground water used for drinking and cooking. The pilot study confirmed that there were no statistically significant differences [between area A and $\mathrm{B}$ ] in either the dietary zinc or iron intake $(\mathrm{p}=0.73$, $\mathrm{p}=0.89$, respectively), or plasma zinc concentration $(p=0.18)$. Groundwater mineral concentrations were compared with data collected previously from community $\mathrm{A}$ and found to be within a comparable range. In both 


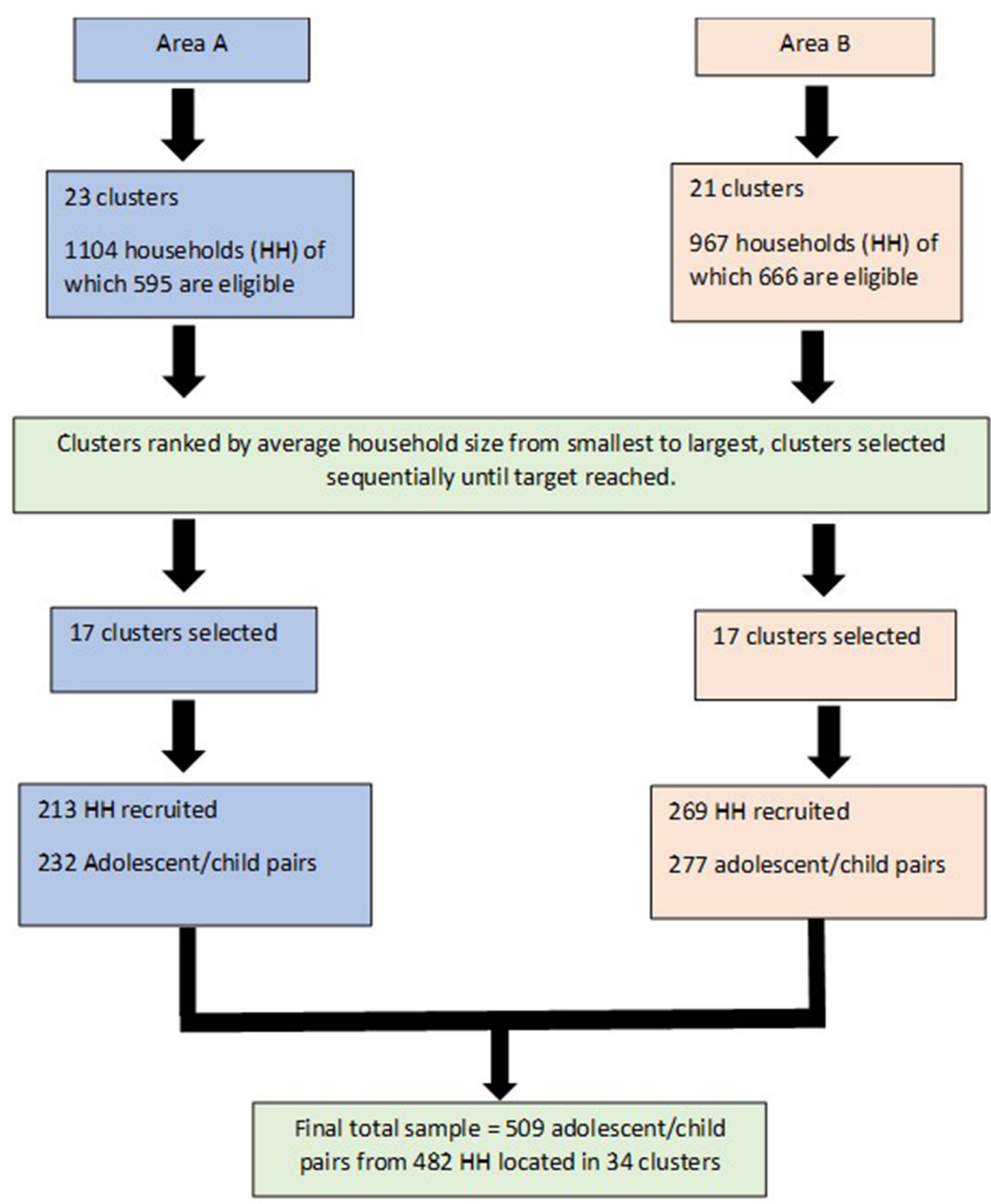

Figure 1 CONSORT flow diagram, schematic overview of the cluster selection procedure. CONSORT, Consolidated Standards of Reporting Trials.

areas, the diet is low in animal-source foods and wheat is the staple. The wheat flour is purchased locally from the market and used for making paratha, chapatti and roti within the household, which are consumed with every meal. Fish is not part of the local diet, and meat is consumed rarely. Approximately $30 \%$ of the WRA have a plasma zinc concentration that falls below the cut-off value of $660 \mu \mathrm{g} / \mathrm{L}$ for adequacy. ${ }^{3}$

The households are located in clusters of variable size, defined by their geographical proximity. Areas A and B comprised 23 and 21 clusters (hamlets), respectively (figure 1). Area A has a health centre, managed and supported by AFPK, that serves as the field team hub and the location for blood sampling and healthcare support during the study. Area B did not have a suitable existing health centre, therefore, some rooms at the AFPK operated school in this community have been coopted for this purpose and alternative classrooms provided elsewhere in the building.
The study field team have undertaken a comprehensive mapping and baseline survey of the catchment area in June-September 2019. The total population is approximately 20000 individuals in approximately 2000 households. Eligibility to participate in the trial was defined as households with at least one unmarried adolescent girl (10-16 years) and one child (1-5 years). Household eligibility was recorded, along with household size (number of individuals cooking and eating together), during the baseline survey and used in the cluster selection and randomisation process described below.

\section{Patient and public involvement}

Cocreation of the research questions and dissemination of findings is embedded within the long-term relationship between the project partners and achieved through ongoing engagement and dialogue with the community and Jirga. Families express concern in community meetings about the health and well-being of their children and understand the relationship between diet and health. 
The community members were not directly involved in the design of this study, however, they are involved in conducting the study. We have employed women from the community to undertake the fortnightly health survey data collection and to report any concerns about the study or other general issues to the study manager. We have employed men from the community to monitor the flour stores and facilitate distribution to participating families. The findings of the study will be communicated to the study participants through the Jirga at an appropriate community forum.

\section{Cluster selection and randomisation procedure}

Clusters were ranked as a function of mean eligible household size (from smallest to largest). Starting from clusters with the smallest average family size, and working down the ranked order, clusters were included until the target sample size was reached. This resulted in an initial selection of 28 clusters (14 from each area) composed of 483 eligible households. The cluster selection was not based on their location; the equal distribution between the two areas was serendipitous, but beneficial in terms of field team logistics enabling an equal spread of field activity between the two areas.

A follow-up survey of all households within the included clusters was undertaken in September 2019 to assess willingness to participate in the study, using a complete enumeration method. The head of each household was approached, and the purpose of the study explained. If the head of the household was willing, the adolescent girl and the mother of the child were approached and the purpose of the study explained, along with the participant information which was provided in written form in the local language (Pushto) and explained verbally. Consent to participate was indicated by signing with initials or an ' $\mathrm{X}$ ' on the consent form. In households where there were two eligible adolescents and children, both pairs were invited to participate. A sample consent form can be found in online supplemental file 1. A total of 19 eligible households declined to participate, therefore, two additional clusters were selected based on the number of eligible households in the cluster and surveyed for willingness to participate. This resulted in a total of 482 participating households from 30 clusters; 15 from area $\mathrm{A}$ and 15 from area $\mathrm{B}$.

During the first data collection phase of the study in November-December 2019 (time point 1) several households withdrew from the study. Two further clusters from each area were selected based on the mean household size. The final number participants were 509 adolescent/ child pairs from 482 households located in 34 clusters (figure 1).

To help ensure comparability of the intervention and control clusters with respect to baseline criteria, the clusters will be matched into pairs according to the average household size of the cluster and the age of the participating adolescent girl. Many investigators conducting community intervention-based trials have shown that matching is a useful mechanism for creating comparable groups at baseline ${ }^{10}$ and mediated good face validity with regard to a balance between trial allocation arms. ${ }^{11}$ Within each pair of clusters, one will be allocated to receive the intervention flour and the other to receive control flour, the allocation being done at random with each cluster having an equal prior probability of being allocated to the intervention treatment. ${ }^{12}$ The random allocation of clusters to either intervention or control will be via a computer-generated software application (Random Allocation Software). Only the study director (MZA), who is not directly involved in the data collection or analysis, will have access to the recorded allocation for each cluster.

The participants and all the research team members, including the principal investigator (PI) and co-PIs (except MZA), field team, laboratory scientists, database manager and data analysts will remain blind to the allocation of the clusters to the intervention or control arm of the study. A data monitoring committee (DMC) will be assigned (from staff within the Clinical Trials Unit at Khyber Medical University (KMU), who are not involved in the study) and will have access to unblinded data from the trial. The DMC will review the interim data analyses including adverse events at three periods during the study and advise the steering committee whether the study needs to be changed, un-blinded or terminated based on these analyses. The three review periods have been identified as follows:

Review period 1: May 2020 (data collected during the baseline and midpoint of phase 1 ).

Review period 2: August 2020 (data collected at the end of phase 1 and preliminary data for phase 2).

Review period 3: October 2020 (data collected at the midpoint of phase 2).

\section{Local production of Zincol-2016 grain for the intervention study}

Forty farmers, with a combined growing area of 100 hectares in close proximity to Peshawar $(25 \mathrm{~km}$ from the study communities), have given consent to grow Zincol-2016 wheat grain for onward use in the effectiveness study with support from Fauji Fertilizer Company (FFC), a collaborating organisation within the BIZIFED2 consortium. Soil samples from each of the fields have been analysed for organic and mineral content by FFC soil testing lab to secure site-specific fertiliser advice for each of the 40 farms according to standard agronomic practice. Farmers have been provided with the Zincol-2016 grain, soil and foliar fertilisers as per this advice. The wheat produced by the farmers will be purchased at a favourable rate. The grain was sown between mid-October and mid-November 2019 and will be closely monitored by a Regional Agronomist of FFC. Zinc foliar spray $(0.1 \%$ elemental zinc; 303 g of $\mathrm{ZnSO}_{4} \cdot \mathrm{H}_{2} \mathrm{O}$ in $100 \mathrm{~L}$ of water used over 0.4 ha of crop) will be applied at booting stage, heading stage and a week after head emergence. Harvest will take place in April/May 2020, and the resulting grain will be milled and provided to families during phase 2 of the study 


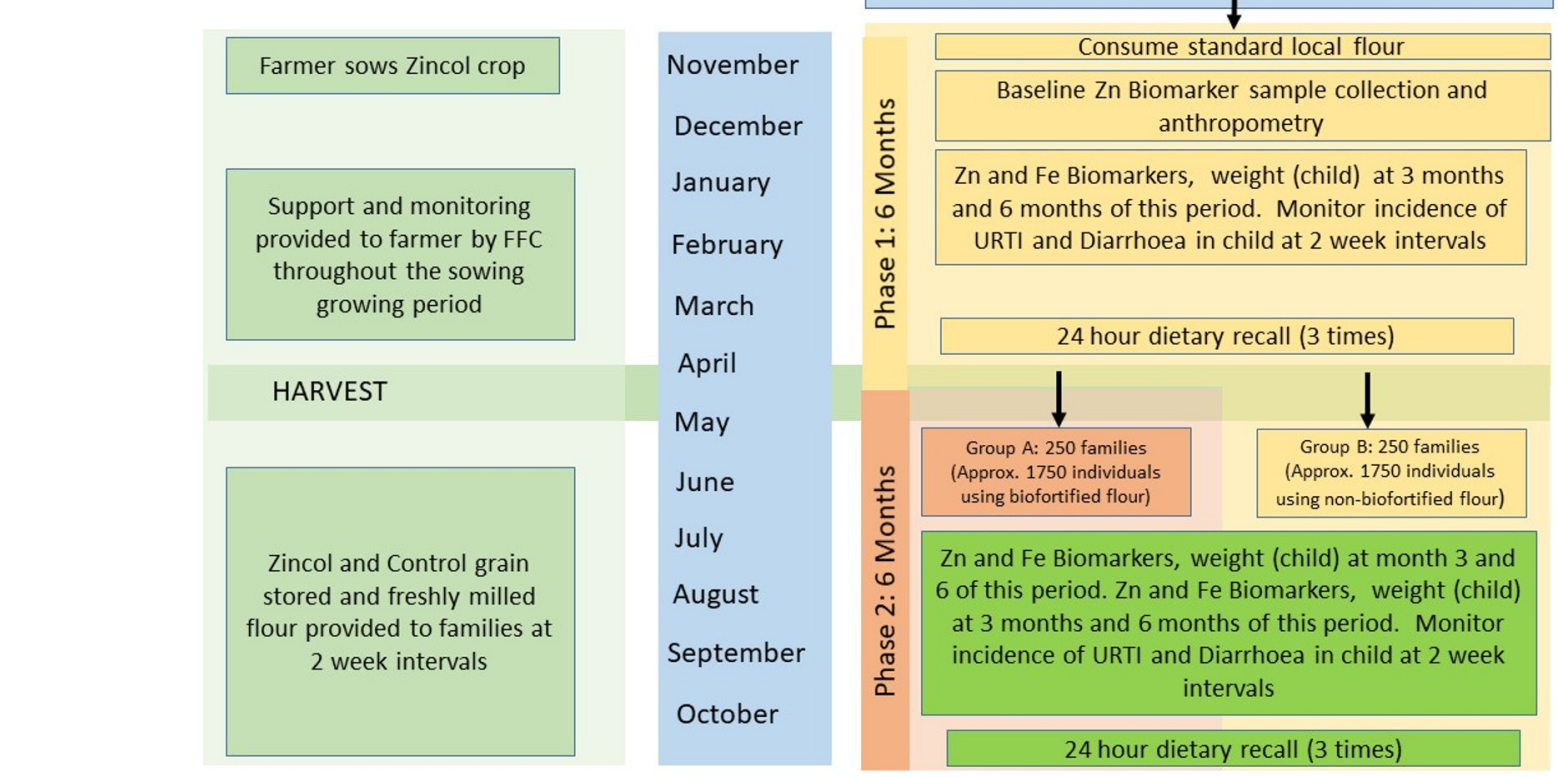

500 adolescent girls and children

Figure 2 Schematic overview of the study design. FFC, Fauji Fertilizer Company; RTI, respiratory tract infection.

(figure 2). Control grain for phase 2 will be purchased from commercial farms in Punjab province, and milled locally for distribution to clusters in the control arm of the study. Prior to the start of phase 2, the zinc content of the control and intervention grain will be determined. The target grain zinc concentrations for the control and intervention grains are 20 and $40 \mathrm{mg} / \mathrm{kg}$, respectively. In the unlikely event that there is no difference in grain $\mathrm{Zn}$ concentrations the trial will be terminated. The iron content of the Zincol-2016 grain and the control grain will be monitored for comparison and interpretation of the iron status biomarkers in the participants.

\section{Study design}

We are conducting a cluster-randomised, double-blind, controlled trial. The study will be undertaken in full compliance with the Consolidated Standards of Reporting Trials (CONSORT) guidelines for cluster randomised analyses. ${ }^{13}$ A cluster randomised trial is necessary due to the community-based nature of the intervention and to reduce the likelihood of contamination, since neighbouring households regularly share flour or consume meals together. ${ }^{14}$ To estimate the sample size to detect a $48 \mu \mathrm{g} / \mathrm{L}$ change in plasma zinc concentration, based on data from the previous trial, the following information was used: (1) a pragmatic and conservative estimate of the likely intraclass correlation coefficient (ICC) taking into account the recommendations of Hemming and Taljaard ${ }^{15}$ for a moderate ICC value (ICC=0.1); (2) similarly a pragmatic estimate of the expected average cluster size based on discussions with the Pakistan field team leader $(n=15)$; (3) to account for unequal cluster sizes an estimate of the coefficient of variation of cluster sizes ( 0.55 was calculated using the equation of Eldridge $\left.e t a^{16}\right)$ taking into account the expected mean cluster size as well as the expected range of cluster sizes, similarly based on discussions with the Pakistan field team; (4) a significance level at $5 \%$ and power of at least $90 \%$; (5) an estimated likely attrition rate of $20 \%$. The sample size that was yielded was $\mathrm{N}=500$. The trial is composed of two 6-month phases, starting on the 4 November 2019. The purpose of phase 1 is to establish baseline values for the outcome measures and the intraindividual variation over a 6 -month period while the participating household are following their usual dietary patterns. During phase 1, all households in the participating clusters will be provided with flour purchased locally, with three data collection points at the start, middle and end of phase 1 . Phase 2 will start in month 7 (May 2020) during which half of the clusters will receive the locally grown biofortified flour and half will receive control flour for a period of 6 months with data collection points at the middle and endpoint of phase 2 (figure 2). Sufficient flour for all the household members (calculated based on the household size, and the flour consumption rate reported during recruitment) will be distributed fortnightly throughout phase 1 and phase 2 , and batches of flour will be sampled to monitor the iron, zinc and phytate content. Participants will collect the flour from a distribution point on presentation of a voucher. Sacks of control and biofortified flour will be identical and labelled using a unique alpha numeric code which does not disclose which type of flour is contained within to the participants but can be used to monitor adherence and fidelity of treatment allocation. Compliance, including the purchase of any addition flour from the market, or any flour received from neighbours will be monitoring during the five data collection time points. 


\section{Outcome measures}

The aim of the randomised controlled trial (RCT) is to assess the impact of consuming biofortified flour on biochemical markers of zinc and iron status in adolescent girls, and functional outcome measures in adolescent girls and children. The outcome measures pertain to the participant not the cluster. The primary outcome measure is plasma zinc concentration, adjusted for inflammatory markers. The method for this has been described in detail previously. ${ }^{7}$ In brief, whole blood samples will be collected into trace element free tubes (BD Vacutainer, BD Diagnostics, Switzerland), centrifuged and the plasma removed within $40 \mathrm{~min}$. Plasma will be stored on ice at the health centre until transfer and storage at $-20^{\circ} \mathrm{C}$ at $\mathrm{KMU}$ within 24 hours. Zinc and other mineral concentrations in the plasma will be measured simultaneously using inductively coupled plasma-mass spectrometry at the University of Nottingham, UK. Seronorm Trace Elements Serum level 1 and 2 (Nycomed Pharma AS, Billingstad, Norway) will be used as the certified reference materials to verify accuracy of the above multielement analyses. Inflammatory markers, alpha 1-acid glycoprotein (AGP) and C reactive protein (CRP) will be measured using commercial kits and Abbott Architect ci8200 automated analyser (Abbott, Abbott Park, Illinois, USA) at Rehman Medical Institute, Peshawar, Pakistan Multigen CRP Vario (Abbott Laboratories) immunoassay kit will used for the immunoturbidimetric determination of CRP in the plasma of the study participants. For immunoturbidimetric estimation of AGP, Quantia A-1- alpha 1-AGP assay kit (Abbott Laboratories) will be used. Manufacturer's instruction would be followed for performing the assays including the use of suggested calibrators and controls. Laboratory quality control performance will be tracked using Westgard rule criteria on the Levey-Jennings chart.

For adjusting plasma zinc concentration for inflammatory markers, recent guidelines from International Zinc Nutrition Consultative Group will be followed. ${ }^{17}$ Briefly, correlation and decile analyses will be performed to understand the magnitude and direction of the association between plasma zinc concentrations, CRP and AGP. Only, if the results of the correlation and decile analysis indicate a clear, negative association between plasma zinc concentrations and CRP and/or AGP, the Biomarkers Reflecting Inflammation and Nutritional Determinants of Anemia regression correction approach will be applied possibly including both CRP and AGP in the regression model.

Secondary outcome measures are as follows:

\section{Additional biomarkers of zinc status}

Measures of DNA damage using the Comet Assay, a novel biomarker of zinc status, will be taken on a random subsample of 100 adolescent participants, at three time points; baseline (end of phase 1), mid, and end of phase 2. Whole blood will be transported from the field location on ice to the laboratory (University of Peshawar) and processed within 2-3 hours according to the previously published protocol. ${ }^{18}$

Hair samples will also be collected from all adolescent participants at all five time points by combing through the hair and collecting those hairs from the comb that has cells from follicle attached to the proximal end of the hair as previously described. ${ }^{7}$ Zinc concentration of hair will be analysed exploiting a novel X-ray fluorescence technique. $^{19}$

\section{Biomarkers of iron status}

Iron status will be assessed using serum transferrin receptor, serum ferritin concentrations. This will be undertaken at the Rehman Medical Institute, Peshawar, Pakistan. To measure soluble transferrin receptor, particle-enhanced immunoturbidimetric assay (Tina quant sTfR, Roche Diagnostics) on a fully mechanised analyzers Cobas 6000 (Roche Diagnostics) will be conducted. Ferritin will be quantified by a chemiluminescent microparticle immunoassay technology based commercial kit (Architect ferritin 7K59, Abbott Laboratories) on by an automated analyzer (Abbott Architect ci8200, Abbott Laboratories, Abbott Park, Illinois, USA), These assays will be performed as per the manufacturer's instruction including the run for recommended controls. Ferritin will be adjusted for inflammation (AGP and CRP) according to the latest guidelines by WHO. ${ }^{20}$

\section{Haematology}

Complete blood count, haematocrit, haemoglobin and mean corpuscular volume will be estimated using standard automated clinical haematology methods at the field site health centre.

\section{Functional indicator of zinc status}

Incidence and duration of diarrhoeal episodes (children only) and respiratory tract infections (children and adolescent girls) will be recorded every 2 weeks throughout the study, through interview with the mother (for the child) and adolescent girl by the female community worker assigned to that cluster.

\section{Anthropometry}

Anthropometric measures of children and adolescent girls will be made at all five data points, including height (or length in the children aged 1-2 years), weight, midupper arm circumference. In addition, waist and hip circumference measures will be made in adolescent girls, and head circumference will be measured in children at all five time points.

\section{Diet analysis}

Overall nutrient intake from the diet and the contribution to total dietary zinc and iron from biofortified flour will be quantified. Dietary nutrient intake will be assessed by multiple pass 24 hours recall. Within each household, the adolescent girl and the mother of the young child will be interviewed by a study nutritionist and portion sizes will be estimated using a locally produced and validated 
food atlas. A total of five recall interviews, three in phase 1 and two in phase 2, will provide a good estimate of typical nutrient intake in both the rainy and the dry seasons. The dietary intake data will be analysed using WinDiets Research Software (2015), Robert Gordon University UK, augmented with data from recent Indian food composition database, ${ }^{21}$ and laboratory analysis of study-specific wheat flour and commonly consumed recipes made by the Pakistan based study nutritionist.

The bioavailability of zinc and iron will be measured from foods made from wheat flour (both fortified and control), including paratha, chapatti and roti breads. Human intestinal Caco-2 cell line, when used together with in vitro digestion, permits the analysis of complex interactions in whole foods, for example, the digestibility of plant cell walls and the release of iron in a form that is available for absorption. ${ }^{22}$ This model will be used to assess zinc and iron availability from control and biofortified (Zincol-2016) wheat lines and food products made from these flours (at King's College London, UK). Mathematical modelling methods combining phytate, iron, and zinc intake will be used to predict absorption levels from the local diet. ${ }^{23}$

\section{Withdrawal and lost to follow-up}

Households and individuals are free to withdraw from the trial at any time. If a household or individual withdraws from the study, their data will remain on file and be used in the final analyses, as indicated on the study consent form.

Households or individuals that move away from the study catchment area will leave the trial and no attempt will be made to follow them up. Any data collected from the household will remain on file and be used in the final analyses.

Throughout the intervention period (November 2019 to November 2020), community engagement events and information sessions will be regularly conducted to deal with any community concerns as they arise and encourage ongoing participation in the study. Any serious community concerns that arise during the intervention period will be addressed and resolved with through the community Jirga system.

\section{Data collection and storage}

Sample analysis will take place in specialist laboratories in the UK and Pakistan. Quality control data (reference materials, detection limits) and full protocols will be reported with the data to ensure that all information is stored together. Several data types will be generated in this project, which will be managed compliant with standard United Kingdom Research and Innovation (UKRI) policies (https://bbsrc.ukri.org/documents/ data-sharing-policy-pdf/). The University of Central Lancashire (UCLan) will be the Data Hub for the project. Data protection and management systems will be clearly communicated to all project partners. UCLan adheres to an Open Data policy and has an open data repository
(UCLan Data). Metadata will be collected in the form of 'read me' files according to the Dublin Core Metadata Initiative. The study will generate personal data from the analysis of biological samples. Participants will be given a unique ID number on recruitment, and this will be used in all data files. The list of names associated with the unique ID numbers will only be known by the project manager and will be stored in a locked filing cabinet. Biological samples will be labelled using the same unique ID numbers. Samples will be shipped to international laboratories for analysis using a secure courier service. Data entry templates (in Excel) will be codeveloped by UCLan and Abaseen Foundation, with variables clearly defined in 'read me' files. Data will be transferred to UCLan in password-protected files using OneDrive. UCLan systems are automatically backed up. Data entry will be checked and cleaned prior to analysis, which will be conducted at UCLan. Data and metadata will be securely stored in a project database and reported on publication.

\section{Statistical analysis plan}

The reporting and presentation of this trial will similarly be in accordance with the CONSORT guidelines for cluster randomised trials, ${ }^{13}$ with the primary comparative analysis being conducted on an intention-to-treat basis. The statistical analysis protocol will be undertaken on both primary and secondary continuous data using an intention to treat basis, whereby participants will be analysed by the arm to which their cluster was randomised. To account for the cluster-based nature of the randomisation process, linear random-effects modelling for the primary and secondary outcomes will be used to compare the two groups. We expect to adjust the models to account for covariate values at baseline. A significance level of 5\% will be adopted, and effectiveness estimation will include both point and $95 \%$ CI estimates.

\section{Ethics and dissemination}

Ethical approval for the research has been granted from the lead University (UCLan) (unique reference number: STEMH 1014), the National Bioethics Committee of Pakistan (NBC-433) and the collaborating institution in Pakistan, KMU (DIR/KMU-EB/BZ/000683). All data collected will be anonymised and stored on secure servers, with data protection protocols followed when information is shared between participating institutions. The final study methods (including any modifications) will be published in peer-reviewed journals, alongside the study outcomes on completion of the data analysis.

Authorship will be restricted to those who have contributed to the design of the study, data collection and analysis, in accordance with the International Committee of Medical Journal Editors guidance. ${ }^{24}$ The protocol used to decide the order of authorship will be published in the paper acknowledgements. In addition to peer-reviewed journals, findings will be disseminated to the scientific community via conference presentations and abstracts. The findings of the study will be communicated to the 
study participants through the village elders at an appropriate community forum. More widely, the AFPK can access some of the most difficult-to-reach communities in KP and the Federally Administered Tribal Area, including through tribal networks, local Jirga and religious leaders. We will work with our contacts in the agriculture sector to exchange knowledge with key actors. For example, the Agricultural Services Department of FFC provides soil, water, fertiliser and plant analysis and recommendation services (in addition to other farmer education/training programmes) to the farming community throughout the country. Project partners and collaborators are well connected to ensure that new evidence can support policy.

A strategic advisory group (SAG), comprised of key international stakeholders, policy-makers and community members, will provide external guidance and help ensure that the project remains relevant to the end-users. The SAG will be consulted by email throughout the 2-year project, particularly during the first 6-month consultation period and will meet by video conference every 6 months.

\section{CONCLUSION}

The RCT described in this protocol paper is part of a larger programme of research that aims to generate data on the effectiveness, acceptability and feasibility of a wheat biofortification strategy for the improvement of micronutrient status in the Pakistan context. Our approach aligns with the Government of Pakistan's Strategy for Improving Nutrition, fundamental to which is the generation of new evidence to inform policy at national and international levels.

\section{Author affiliations}

${ }^{1}$ UCLan Research Centre for Global Development, University of Central Lancashire,

Preston, UK

${ }^{2}$ Department of Pulmonology, Rehman Medical Institute, Peshawar, Khyber Pakhtunkhwa, Pakistan

${ }^{3}$ Institute of Basic Medical sciences, Khyber Medical University, Peshawar, Pakistan ${ }^{4}$ School of Biosciences, University of Nottingham, Sutton Bonington Campus, Nottingham, UK

${ }^{5}$ Department of Population Health, London School of Hygiene and Tropical Medicine, London, UK

${ }^{6}$ Institute of Health Professions Education and Research, Khyber Medical University, Peshawar, Pakistan

${ }^{7}$ Research and Development, Fauji Fertilizer Co Ltd, Rawalpindi, Punjab, Pakistan

${ }^{8}$ Inorganic Chemistry, Centre for Environmental Geochemistry, British Geological Survey, Nottingham, UK

${ }^{9}$ Nutritional Sciences, Kings College London, London, UK

\section{Twitter Nicola M Lowe @NicolaLoweZinc}

Acknowledgements The authors gratefully acknowledge the Abaseen Foundation Pakistan for use of the Health Centres and facilitating access to the community and Abaseen Foundation UK for diligent oversight of the transfer of funds. We sincerely thank the community members and elders who have shared their thoughts with us and worked with us to identify the most important challenges to health and wellbeing that we can work on together to solve. We are grateful to the AF PK field team, led by Muhammad Javaid for helping us get this ambitious study underway. We acknowledge the support of our UK research assistants Dr Swarnim Gupta and Anna Brazier in the smooth running of this study and to Swarnim for assistance in the preparation of this manuscript.We are grateful for the in-kind contribution of
Fauji Fertilizer Company (FFC) for support with crop management and production. The authors would like to thank the members of our Study Advisory Group, chaired by Professor Harry McArdle, for helpful discussions. We have followed the 'sequence-determines-credit' approach for the order of the authors listed. The sequence of authors reflects the declining importance of their contribution to this element of the BIZIFED2 programme.

Contributors NML (PI) and the co-PIs, MZ, MRB, MHZ, UM, MJK (deceased June 2019), EJMJ, HO, ELA, RML, PAS, JKS, EHB, SDY and VHM conceived the research questions and designed the study protocol. SF is overseeing the training of the study nutritionists, and the diet data collection. All authors have read and approved this manuscript.

Funding This work is supported by BBSRC Global Challenges Research Fund, Grant Number BB/S013989/1. The trial is sponsored by the University of Central Lancashire and can be contacted through Professor NML.

Competing interests None declared.

Patient consent for publication Not required.

Provenance and peer review Not commissioned; externally peer reviewed.

Supplemental material This content has been supplied by the author(s). It has not been vetted by BMJ Publishing Group Limited (BMJ) and may not have been peer-reviewed. Any opinions or recommendations discussed are solely those of the author(s) and are not endorsed by BMJ. BMJ disclaims all liability and responsibility arising from any reliance placed on the content. Where the content includes any translated material, BMJ does not warrant the accuracy and reliability of the translations (including but not limited to local regulations, clinical guidelines, terminology, drug names and drug dosages), and is not responsible for any error and/or omissions arising from translation and adaptation or otherwise.

Open access This is an open access article distributed in accordance with the Creative Commons Attribution Non Commercial (CC BY-NC 4.0) license, which permits others to distribute, remix, adapt, build upon this work non-commercially, and license their derivative works on different terms, provided the original work is properly cited, appropriate credit is given, any changes made indicated, and the use is non-commercial. See: http://creativecommons.org/licenses/by-nc/4.0/.

\section{ORCID iDs}

Nicola M Lowe http://orcid.org/0000-0002-6934-2768

Victoria Hall Moran http://orcid.org/0000-0003-3165-4448

\section{REFERENCES}

1 Allen L, de Benoist B, Dary O, et al. Guidelines on food fortification with micronutrients. Geneva: World Health Organization and Food and Agricultural Organization of the United Nations, 2006.

2 UNICEF. National Nutrition Survey 2018. Key Findings Report. In: Nutrition wing Ministry of health services, regulation and coordination, Government of Pakistan, ed. Pakistan, 2019.

3 Brown KH, Rivera JA, et al, International Zinc Nutrition Consultative Group (IZiNCG). International Zinc Nutrition Consultative Group (IZiNCG) technical document \#1 Assessment of the risk of zinc deficiency in populations and options for its control. Food Nutr Bull 2004;25:S99-203.

4 Mayo-Wilson E, Junior JA, Imdad A, et al. Zinc supplementation for preventing mortality, morbidity, and growth failure in children aged 6 months to 12 years of age. Cochrane Database Syst Rev 2014;5:CD009384.

5 Kassebaum NJ, Jasrasaria R, Naghavi M, et al. A systematic analysis of global anemia burden from 1990 to 2010. Blood 2014;123:615-24.

6 Ansari N, Mehmood R, Gazdar H. Going against the grain of optimism: flour fortification in Pakistan. IDS Bull 2018;49:57-71.

7 Lowe NM, Khan MJ, Broadley MR, et al. Examining the effectiveness of consuming flour made from agronomically biofortified wheat (Zincol-2016/NR-421) for improving Zn status in women in a lowresource setting in Pakistan: study protocol for a randomised, double-blind, controlled cross-over trial (BiZiFED). BMJ Open 2018;8:e021364.

8 Ohly H, Broadley MR, Joy EJM, et al. The BiZiFED project: biofortified zinc flour to eliminate deficiency in Pakistan. Nutr Bull 2019;44:60-4.

9 Schroeder D, Adhiambo J, Altare C, et al. Global code of conduct for research in resource-poor settings. Available: https://www. globalcodeofconduct.org/ [Accessed 08/04/2020].

10 Campbell MJ, Donner A, Klar N. Developments in cluster randomized trials and statistics in medicine. Stat Med 2007;26:2-19. 
11 Donner A, Klar N. Pitfalls of and controversies in cluster randomization trials. Am J Public Health 2004;94:416-22.

12 Grosskurth H, Mosha F, Todd J, et al. A community trial of the impact of improved sexually transmitted disease treatment on the HIV epidemic in rural Tanzania: 2. baseline survey results. AIDS 1995;9:927-34.

13 Campbell MK, Elbourne DR, Altman DG, et al. CONSORT statement: extension to cluster randomised trials. BMJ 2004;328:702-8.

14 Torgerson DJ. Contamination in trials: is cluster randomisation the answer? BMJ 2001;322:355-7.

15 Hemming K, Taljaard M. Sample size calculations for stepped wedge and cluster randomised trials: a unified approach. J Clin Epidemiol 2016;69:137-46.

16 Eldridge SM, Ashby D, Kerry S. Sample size for cluster randomized trials: effect of coefficient of variation of cluster size and analysis method. Int J Epidemiol 2006;35:1292-300.

17 International Zinc Nutrition Consultative Group. Adjusting plasma or serum zinc concentrations for inflammation IZiNCG technical brief No. 10. IZiNCG, 2020. Available: http://www.izincg.org [Accessed 21 Sep 2020].
18 Khisroon M, Khan A, Ullah U, et al. Bio-monitoring of DNA damage in matchstick industry workers from Peshawar Khyber Pakhtunkhwa, Pakistan. Int J Occup Environ Health 2018;24:126-33.

19 Fleming DEB, Bennett SR, Frederickson CJ. Feasibility of measuring zinc in human nails using portable X-ray fluorescence. J Trace Elem Med Biol 2018;50:609-14.

20 World Health Organization. Who guideline on use of ferritin concentrations to assess iron status in individuals and populations. Geneva: World Health Organization, 2020.

21 Longvah T, Ananthan R, Bhaskarachary K, et al. Indian Food Composition Tables. In: Department of health research, Ministry of health and family welfare, Government of India, ed. Hyderabad, 2017.

22 Sandberg A-S. The use of caco-2 cells to estimate fe absorption in humans--a critical appraisal. Int J Vitam Nutr Res 2010;80:307-13.

23 Miller LV, Krebs NF, Hambidge KM. A mathematical model of zinc absorption in humans as a function of dietary zinc and phytate. $J$ Nutr 2007;137:135-41.

24 International Committee of Medical Journal Editors. Defining the role of authors and contributors. Available: http://www.icmje.org/ recommendations/browse/roles-and-responsibilities/defining-therole-of-authors-and-contributors.html [Accessed 3 April 2020]. 